\title{
Agro-ecological and technological quality of some apples
}

\author{
ANTON SOTIROV ${ }^{1}$, NIKOLAY GLAVEV ${ }^{1}$, DIMITAR SOTIROV ${ }^{2}$, STANISLAVA DIMITROVA $^{2}$, \\ NIKOLA PISTALOV ${ }^{3}$, VIKTOR SOTIROV ${ }^{3}$, KRASIMIR SOTIROV $^{3}$ \\ ${ }^{1}$ „Velanto Group“ Ltd., Kyustendil \\ ${ }^{2}$ Institute of Agriculture, Kyustendil \\ ${ }^{3}$ Environmental Association "Club Vanguard", Kyustendil
}

BULGARIA

\begin{abstract}
The aim of the study is to investigate cloudy apple juices, produced from 83 apple varieties, grown by the Institute of Agriculture in town of Kyustendil, Bulgaria for their main ecological and technological parameters, in view of their safety for the population and the possibilities for their use as raw material in the food industry. The study found, that the apples are environmentally friendly for the studied parameters. Technologically, there are varieties with the highest juice yields (over 70\%) - Defloga, Ginger Gold, Gold Rush, Granny Smith, and Melrose. The most tasty and colored juices of fresh fruit and fruit flour are separated from the Florina, Free Redstar, Ginger Gold, Gold Rush, Golden Delicious, and Granny Smith. Some practically do not give juice and material for fruit flour with the applied method $100 \%$ cold-pressed apple juice, but they produce puree with high quality as the Belgolden, Braeburn, Charden, and Red Delicious. There were observed very stable approximate ratios between Conductivity, Total Dissolved Solids, and Salt content in the juices as follows: Cond./TDS=1.5, TDS/Salt=1.3, Cond./Salt=2. Retio between Total Sugar Content and Total Acidity $\mathrm{Brix} / \mathrm{pH}=3-5$, as the most tasty and with good color juices have ratio $\mathrm{Brix} / \mathrm{pH}=4$.
\end{abstract}

Key-Words: - agroecology, technology, apples, juices, chemical, quality

Received: September 15, 2020. Revised: January 11, 2021. Accepted: January 27, 2021. Published: February 1, 2021.

\section{Introduction}

Apple (Malus domestica Borkh.) is the main raw material for the food industry and cooking in terms of fruit. It is the main raw material in a wide range of productions in the food industry. It is used for producing of cloudy and clear juice, syrups, concentrates, nectars, purees, wine, vinegar, cider, malic acid, pectin, fruit flours, dried fruits, compotes, marmalades and more. It is an indispensable fruit for direct consumption, canning, as well as in cooking and confectionery for various dishes.

The aim of the study is to be analyzed 83 of the popular apples, grown by the Institute of Agriculture in town of Kyustendil, Bulgaria for their main agro-environmental and technological parameters, in view of their safety for the population and the possibilities for their use as raw material for the production of cold-pressed juices in the canning industry.

\section{Methods}

\subsection{Agro-ecological parameters}

The total radiation background, the radioactivity of the fruit and the juice obtained from them were measured with a Geiger counter "Radex" RD1503 in the microsievert per hour $(\mu \mathrm{Sv} / \mathrm{h})$. Four consecutive measurements of radioactivity were performed of each apple variety and their juices, without separation of the individual types of radiation, followed by averaging of the value, performed automatically.

Nitrate $\mathrm{NO}_{3}^{-} \mathrm{mg} / \mathrm{l}$ and nitrite $\mathrm{NO}_{2}^{-} \mathrm{mg} / \mathrm{l}$ contents in the obtained juice were determined by semi-quantitative colorimetric analysis with test strips with a range of 0-10-25-50-100-250-500 $\mathrm{mg} / \mathrm{l}$.

The arsenic As content was measured by semi-quantitative colorimetric analysis with a set of test strips with a range of 0,000-0,005-0,010-0,025$0,05-0,1-0,25-0,5 \mathrm{mg} / \mathrm{l}$ and malonic acid reagent.

For determination of the zinc $\mathrm{Zn}$ content, a semi-quantitative colorimetric analysis was applied with a set of test strips with a range of 0-4-10-20-50 $\mathrm{mg} / \mathrm{l}$ and sodium hydroxide reagent.

Manganese Mn was also determined by semi-quantitative colorimetric analysis with a set of test strips in the range 0-2-5-20-50-100 $\mathrm{mg} / \mathrm{l}$ and sodium hydroxide reagent.

The content of lead $\mathrm{Pb}$ was established by semi-quantitative colorimetric analysis - Blei-Test, with a set of test strips with a range of 0-20-40-100200-500 $\mathrm{mg} / \mathrm{l}$ and reagent rhodic acid.

Sulfates $\mathrm{SO}_{4}{ }^{2-}$ were measured by semiquantitative colorimetric analysis with test strips with a range of 0-200-400-800-1200-1600 mg/l. 
$\mathrm{SO}_{3}{ }^{2-}$ sulfites were also measured by semiquantitative colorimetric analysis with test strips in the range $0-10-40-80-180-400 \mathrm{mg} / \mathrm{l}$.

\subsection{Technological parameters}

Portable digital instruments were used as follow: Brix refractometer MA871" to measure the total sugar content by Brix (\%),"SensoDirect 150" apparatus and "Waterproof IP57" tester for determination of total acidity $(\mathrm{pH})$, electrical conductivity $(\mu \mathrm{S})$, total dissolved solids ( $\mathrm{ppm})$, total salt content (ppm). The glucose content in the apples was measured with a CodeFree titration glucometer (range 0,6-33,3 mmol/l).

The yield (\%) was calculated, ie. the ability of apples to release juice during cold pressing of the fruit, as the measurements of the weight of the apples and the juice released during pressing were performed with a laboratory analytical balance.

Sensory testing was performed also, such as taste, aroma, color and turbidity of the juice and the separated fruit puree.

Apple juice $(100 \%)$ is obtained by the method of cold pressing with a single-shaft juicer Star Light SJB-150 R, unpasteurized, without additives.

The topic is developed in different ways in the scientific literature, as $[1,2,3]$ present research related to the protection of apples from radiation during their storage. Information about possible sources of radioactive contamination is given by [4]. Data on the old apple in Kyustendil region, Bulgaria give $[5,6]$. Extensive analysis of apples as a fruit is presented [7], and [8,9] make a description and classification of apple juices. Other authors perform a qualitative analysis of apple juices $[10,11]$. Regarding the chemical composition of apples and their juices, the topic is well developed, for example, such data give $[12,13,14,15,16,17$, $18,19,20,21,22,23,24,25,26]$ and others.

\section{Results}

Table 1 Measured almost constant agro-ecological parameters for all juices and purees of different apple varieties

\begin{tabular}{|c|c|c|c|c|c|c|c|c|c|}
\hline 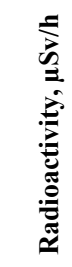 & 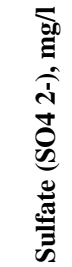 & 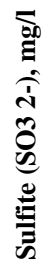 & 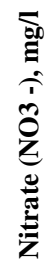 & 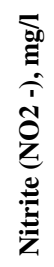 & $\begin{array}{l}\sigma_{00} \\
\Xi \\
\dot{\Xi}\end{array}$ & 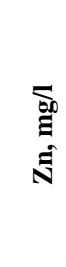 & 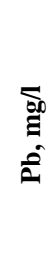 & 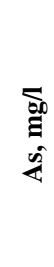 & 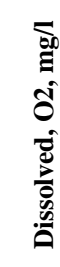 \\
\hline $\begin{array}{c}0.10- \\
0.18\end{array}$ & $\begin{array}{l}200- \\
300\end{array}$ & 0 & 0 & 0 & $1-2$ & $0-1$ & 0 & 0 & $\begin{array}{c}0.4- \\
0.6\end{array}$ \\
\hline
\end{tabular}

Table 2 Measured technological parameters of the juices and purees of different apple varieties

\begin{tabular}{|c|c|c|c|c|c|c|c|}
\hline № & $\begin{array}{r}\text { Juice } \\
\text { parameters }\end{array}$ & 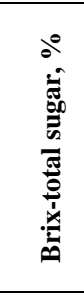 & 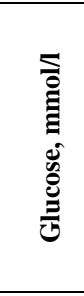 & 承 & 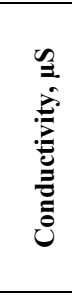 & 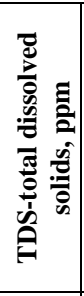 & 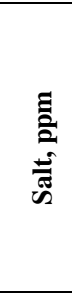 \\
\hline 1 & Aivanija & 12.10 & $>33.3$ & 5.37 & 1726 & 1154 & 866 \\
\hline 2 & $\begin{array}{l}\text { Winter } \\
\text { Banana }\end{array}$ & 18.50 & $>33.3$ & 3.45 & 2180 & 1450 & 1110 \\
\hline 3 & Belgolden & 10.10 & $>33.3$ & 3.06 & 1962 & 1313 & 995 \\
\hline 4 & $\begin{array}{l}\text { Berner } \\
\text { Rosenapfel }\end{array}$ & 7.60 & $>33.3$ & 3.31 & 4180 & 2800 & 226 \\
\hline 5 & Besapara & 11.40 & $>33.3$ & 3.39 & 187 & 138 & 98 \\
\hline 6 & Braeburn & 10.40 & $>33.3$ & 3.30 & 2000 & 1350 & 1030 \\
\hline 7 & Buhavitsa & 11.20 & $>33.3$ & 2.65 & 1780 & 1191 & 897 \\
\hline 8 & Charden & 12.40 & $>33.3$ & 3.07 & 3230 & 2170 & 1710 \\
\hline 9 & COOP 10 & 9.90 & $>33.3$ & 3.66 & 763 & 509 & 371 \\
\hline 10 & Defloga & 10.70 & $>33.3$ & 3.33 & 1472 & 986 & 734 \\
\hline 11 & Democrat & 13.20 & $>33.3$ & 3.68 & 2070 & 105 & 1380 \\
\hline 12 & Elegia & 13.30 & $>33.3$ & 3.31 & 751 & 629 & 335 \\
\hline 13 & Erwin Bauer & 14.30 & $>33.3$ & 2.97 & 1462 & 978 & 729 \\
\hline 14 & Florina & 11.70 & 26.9 & 2.9 & 1391 & 935 & 699 \\
\hline 15 & Free Redstar & 14.10 & $>33.3$ & 5.28 & 1208 & 808 & 584 \\
\hline 16 & Freedom & 11.80 & $>33.3$ & 2.85 & 2190 & 1460 & 1120 \\
\hline 17 & Gala Mondial & 10.10 & $>33.3$ & 3.53 & 2680 & 1790 & 1380 \\
\hline 18 & Ginger Gold & 11.20 & $>33.3$ & 2.92 & 1515 & 1020 & 763 \\
\hline 19 & Gloster 69 & 11.30 & $>33.3$ & 3.91 & 1912 & 1277 & 967 \\
\hline 20 & $\begin{array}{l}\text { Gold } \\
\text { Milenium }\end{array}$ & 12.00 & $>33.3$ & 3.25 & 1671 & 1116 & 839 \\
\hline 21 & Gold Rush & 13.60 & $>33.3$ & 3.48 & 2190 & 1460 & 1110 \\
\hline 22 & $\begin{array}{l}\text { Golden } \\
\text { Delicious }\end{array}$ & 11.30 & $>33.3$ & 3.26 & 1799 & 1204 & 908 \\
\hline 23 & $\begin{array}{l}\text { Golden } \\
\text { Resistance }\end{array}$ & 13.10 & $>33.3$ & 2.88 & 738 & 495 & 357 \\
\hline
\end{tabular}




\begin{tabular}{|c|c|c|c|c|c|c|c|}
\hline 24 & $\begin{array}{l}\text { Golden } \\
\text { Smoothee }\end{array}$ & 12.20 & $>33.3$ & 3.25 & 2000 & 1340 & 1016 \\
\hline 25 & $\begin{array}{l}\text { Golden } \\
\text { Winter } \\
\text { Pearmain } \\
\end{array}$ & 11.50 & $>33.3$ & 2.92 & 1806 & 1211 & 914 \\
\hline 26 & Gorana & 15.30 & $>33.3$ & 3.33 & 4040 & 2180 & 2700 \\
\hline 27 & $\begin{array}{l}\text { Granny } \\
\text { Smith }\end{array}$ & 11.50 & $>33.3$ & 2.94 & 1250 & 685 & 374 \\
\hline 28 & $\begin{array}{l}\text { Green } \\
\text { Knyajevska }\end{array}$ & 11.20 & $>33.3$ & 2.78 & 1973 & 1323 & 1004 \\
\hline 29 & Hybrid 1/3 & 15.90 & $>33.3$ & 4.02 & 1413 & 942 & 702 \\
\hline 30 & Hybrid 1/37 & 10.90 & $>33.3$ & 3.87 & 1972 & 1321 & 1003 \\
\hline 31 & Hybrid 2/14 & 11.60 & $>33.3$ & 2.72 & 1992 & 1323 & 1004 \\
\hline 32 & Hybrid 2/28 & 11.10 & 26.7 & 3.00 & 1908 & 1277 & 969 \\
\hline 33 & Hybrid 2/30 & 13.00 & 27.1 & 3.81 & 507 & 765 & 370 \\
\hline 34 & Hybrid 2/4 & 13.00 & $>33.3$ & 3.00 & 3760 & 2520 & 2020 \\
\hline 35 & Hybrid 2/8 & 11.70 & $>33.3$ & 3.15 & 1459 & 979 & 724 \\
\hline 36 & Hybrid 6 & 12.00 & $>33.3$ & 3.70 & 3820 & 2560 & 2040 \\
\hline 37 & Hybrid 7 & 10.30 & $>33.3$ & 2.97 & 1911 & 1281 & 971 \\
\hline 38 & Hybrid 8/22 & 13.10 & $>33.3$ & 3.11 & 1901 & 1276 & 968 \\
\hline 39 & Hybrid 8/35 & 13.80 & $>33.3$ & 2.84 & 1460 & 982 & 730 \\
\hline 40 & Hybrid 9 & 14.10 & $>33.3$ & 3.30 & 1833 & 1226 & 925 \\
\hline 41 & Hybrid 9/36 & 12.50 & 25.3 & 3.47 & 3770 & 2520 & 2010 \\
\hline 42 & $\begin{array}{l}\text { Hybrid } \\
\text { Pinova x Fuji }\end{array}$ & 14.00 & $>33.3$ & 3.97 & 1914 & 1282 & 971 \\
\hline 43 & Idagold & 11.30 & $>33.3$ & 2.84 & 1135 & 761 & 558 \\
\hline 44 & Jonagold & 13.50 & $>33.3$ & 2.60 & 712 & 477 & 345 \\
\hline 45 & Kadunka & 13.10 & $>33.3$ & 3.45 & 3850 & 2580 & 2070 \\
\hline 46 & Karastoyanka & 11.50 & $>33.3$ & 3.07 & 1120 & 741 & 548 \\
\hline 47 & $\begin{array}{l}\text { Landsberger } \\
\text { Renette }\end{array}$ & 13.90 & $>33.3$ & 2.90 & 2830 & 1890 & 1470 \\
\hline 48 & Liberty & 15.00 & $>33.3$ & 3.43 & 4450 & 2980 & 2000 \\
\hline 49 & Marlena & 11.60 & $>33.3$ & 3.50 & 2000 & 1340 & 1020 \\
\hline 50 & Martinika & 13.90 & $>33.3$ & 3.20 & 2120 & 1410 & 1070 \\
\hline
\end{tabular}

\begin{tabular}{|c|c|c|c|c|c|c|c|}
\hline 51 & Melrose & 10.30 & $>33.3$ & 3.31 & 1379 & 933 & 695 \\
\hline 52 & $\begin{array}{l}\text { Mollie's } \\
\text { Delicious }\end{array}$ & 11.00 & $>33.3$ & 2.59 & 2620 & 1740 & 1340 \\
\hline 53 & Mutsu & 10.60 & $>33.3$ & 2.91 & 555 & 373 & 271 \\
\hline 54 & $\begin{array}{l}\text { Ovcha } \\
\text { Mutsuna }\end{array}$ & 13.30 & $>33.3$ & 2.52 & 886 & 592 & 430 \\
\hline 55 & Pasific Rose & 12.90 & $>33.3$ & 3.60 & 1106 & 743 & 546 \\
\hline 56 & Pink Lady & 12.60 & $>33.3$ & 2.78 & 905 & 605 & 441 \\
\hline 57 & Pinova & 11.60 & $>33.3$ & 2.91 & 1857 & 1244 & 941 \\
\hline 58 & Priam & 10.50 & $>33.3$ & 5.30 & 1893 & 1260 & 965 \\
\hline 59 & Prima & 10.50 & $>33.3$ & 3.1 & 727 & 664 & 478 \\
\hline 60 & Reandra & 11.10 & $>33.3$ & 2.77 & 1427 & 964 & 710 \\
\hline 61 & Red Chief & 13.22 & $>33.3$ & 5.34 & 1628 & 1090 & 816 \\
\hline 62 & Red Delicios & 11.40 & $>33.3$ & 3.70 & 2010 & 1350 & 1030 \\
\hline 63 & $\begin{array}{l}\text { Reinette du } \\
\text { Canada }\end{array}$ & 12.20 & $>33.3$ & 3.25 & 3690 & 2470 & 1970 \\
\hline 64 & Renora & 12.60 & $>33.3$ & 2.84 & 2820 & 1880 & 1470 \\
\hline 65 & Rosana & 9.80 & $>33.3$ & 3.28 & 1661 & 1112 & 836 \\
\hline 66 & Rosemary & 11.70 & $>33.3$ & 3.62 & 2140 & 1430 & 1090 \\
\hline 67 & Rubinola & 14.60 & $>33.3$ & 3.90 & 1160 & 778 & 574 \\
\hline 68 & Sansa & 12.80 & $>33.3$ & 3.81 & 1415 & 950 & 707 \\
\hline 69 & Sekai Ichi & 13.00 & $>33.3$ & 3.42 & 3850 & 2570 & 2000 \\
\hline 70 & Siyana & 13.90 & $>33.3$ & 3.38 & 410 & 272 & 195 \\
\hline 71 & Skrinyanka & 11.90 & $>33.3$ & 3.02 & 2160 & 1400 & 1090 \\
\hline 72 & Super Chief & 10.30 & $>33.3$ & 3.98 & 2090 & 1390 & 1050 \\
\hline 73 & Teser T219 & 12.30 & $>33.3$ & 3.49 & 4030 & 2790 & 2180 \\
\hline 74 & Tetovka & 9.64 & $>33.3$ & 2.85 & 1595 & 1069 & 799 \\
\hline 75 & Topaz & 16.50 & $>33.3$ & 4.01 & 2010 & 1350 & 1030 \\
\hline 76 & Tsiganka & 12.90 & $>33.3$ & 2.87 & 1795 & 1202 & 904 \\
\hline 77 & Wellington & 10.20 & $>33.3$ & 2.72 & 461 & 287 & 189 \\
\hline 78 & $\begin{array}{l}\text { White } \\
\text { Kandile }\end{array}$ & 13.31 & $>33.3$ & 3.64 & 4400 & 2940 & 2380 \\
\hline 79 & $\begin{array}{l}\text { White Winter } \\
\text { Calville }\end{array}$ & 13.70 & $>33.3$ & 3.22 & 3990 & 2670 & 2150 \\
\hline 80 & $\begin{array}{l}\text { Wild apple } \\
\text { plant }\end{array}$ & 13.20 & $>33.3$ & 2.98 & 1530 & 1058 & 789 \\
\hline 81 & $\begin{array}{l}\text { Winter } \\
\text { Lemon }\end{array}$ & 9.80 & $>33.3$ & 3.34 & 4170 & 2790 & 2250 \\
\hline 82 & Wiygik & 9.70 & $>33.3$ & 2.77 & 1476 & 988 & 736 \\
\hline
\end{tabular}




\begin{tabular}{|l|l|l|l|l|l|l|l|}
83 & $\begin{array}{l}\text { Yellow } \\
\text { Bellefleur }\end{array}$ & 10.30 & $>33.3$ & 2.74 & 4300 & 2800 & 2330 \\
\hline
\end{tabular}

Table 3 Sensory testing of the juices and purees

\begin{tabular}{|c|c|c|c|c|c|c|}
\hline № & $\begin{array}{l}\text { Juice } \\
\text { parameters } \\
\text { Apple } \\
\text { varieties }\end{array}$ & $\frac{\grave{\partial}}{\dot{\theta}}$ & $\stackrel{\Xi}{:}$ & 苞 & 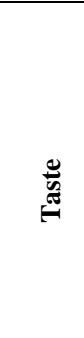 & 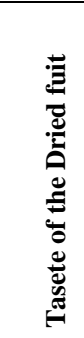 \\
\hline 1 & Aivanija & brown & puree & $\begin{array}{l}\text { dried } \\
\text { fruit }\end{array}$ & sweet & sweet \\
\hline 2 & $\begin{array}{l}\text { Winter } \\
\text { Banana }\end{array}$ & yellow & puree & $\begin{array}{l}\text { fresh } \\
\text { fruit }\end{array}$ & $\begin{array}{l}\text { sweet } \\
\text {-acid }\end{array}$ & $\begin{array}{c}\text { sweet- } \\
\text { acid }\end{array}$ \\
\hline 3 & Belgolden & brown & puree & $\begin{array}{l}\text { dried } \\
\text { fruit }\end{array}$ & $\begin{array}{l}\text { sweet } \\
\text {-acid }\end{array}$ & sweet \\
\hline 4 & $\begin{array}{l}\text { Berner } \\
\text { Rosenapfel } \\
\end{array}$ & yellow & puree & $\begin{array}{l}\text { fresh } \\
\text { fruit }\end{array}$ & $\begin{array}{c}\text { tastele } \\
\text { ss }\end{array}$ & sweed \\
\hline 5 & Besapara & brown & puree & $\begin{array}{l}\text { dried } \\
\text { fruit }\end{array}$ & $\begin{array}{l}\text { sweet } \\
\text {-acid }\end{array}$ & $\begin{array}{c}\text { sweet- } \\
\text { acid }\end{array}$ \\
\hline 6 & Braeburn & brown & puree & $\begin{array}{l}\text { dried } \\
\text { fruit }\end{array}$ & $\begin{array}{l}\text { sweet } \\
\text {-acid }\end{array}$ & $\begin{array}{c}\text { sweet- } \\
\text { acid }\end{array}$ \\
\hline 7 & Buhavitsa & $\begin{array}{l}\text { dark- } \\
\text { green }\end{array}$ & high & $\begin{array}{l}\text { dried } \\
\text { fruit }\end{array}$ & $\begin{array}{l}\text { acid- } \\
\text { sweet }\end{array}$ & $\begin{array}{c}\text { sweet- } \\
\text { acid }\end{array}$ \\
\hline 8 & Charden & brown & puree & $\begin{array}{l}\text { dried } \\
\text { fruit }\end{array}$ & sweet & $\begin{array}{c}\text { sweet- } \\
\text { acid }\end{array}$ \\
\hline 9 & COOP 10 & yellow & puree & $\begin{array}{c}\text { unide } \\
\text { ntifie } \\
\text { d }\end{array}$ & $\begin{array}{l}\text { tastele } \\
\text { ss }\end{array}$ & sweet \\
\hline 10 & Defloga & brown & high & $\begin{array}{l}\text { dried } \\
\text { fruit }\end{array}$ & $\begin{array}{l}\text { sweet } \\
\text {-acid }\end{array}$ & $\begin{array}{l}\text { acid- } \\
\text { sweet }\end{array}$ \\
\hline 11 & Democrat & brown & hign & $\begin{array}{l}\text { dried } \\
\text { fruit }\end{array}$ & $\begin{array}{l}\text { sweet } \\
\text {-acid }\end{array}$ & $\begin{array}{c}\text { sweet- } \\
\text { acid }\end{array}$ \\
\hline 12 & Elegia & yellow & puree & $\begin{array}{l}\text { fresh } \\
\text { fruit }\end{array}$ & sweet & sweet \\
\hline 13 & $\begin{array}{l}\text { Erwin } \\
\text { Bauer }\end{array}$ & $\begin{array}{l}\text { yellow- } \\
\text { green }\end{array}$ & high & $\begin{array}{l}\text { fresh } \\
\text { fruit }\end{array}$ & $\begin{array}{l}\text { acid- } \\
\text { sweet }\end{array}$ & $\begin{array}{c}\text { sweet- } \\
\text { acid }\end{array}$ \\
\hline 14 & Florina & $\begin{array}{l}\text { yellow } \\
\text { brown }\end{array}$ & high & $\begin{array}{l}\text { fresh } \\
\text { fruit }\end{array}$ & $\begin{array}{l}\text { sweet } \\
\text {-acid }\end{array}$ & $\begin{array}{c}\text { sweet- } \\
\text { acid }\end{array}$ \\
\hline 15 & $\begin{array}{l}\text { Free } \\
\text { Redstar }\end{array}$ & yellow & hign & $\begin{array}{l}\text { fresh } \\
\text { fruit }\end{array}$ & sweet & sweet \\
\hline 16 & Freedom & brown & high & $\begin{array}{l}\text { dried } \\
\text { fruit }\end{array}$ & $\begin{array}{l}\text { sweet } \\
\text {-acid }\end{array}$ & sweet \\
\hline 17 & $\begin{array}{l}\text { Gala } \\
\text { Mondial }\end{array}$ & $\begin{array}{l}\text { brown- } \\
\text { green }\end{array}$ & high & $\begin{array}{l}\text { dried } \\
\text { fruit }\end{array}$ & $\begin{array}{l}\text { sweet } \\
\text {-acid }\end{array}$ & $\begin{array}{l}\text { acid- } \\
\text { sweet }\end{array}$ \\
\hline
\end{tabular}

\begin{tabular}{|c|c|c|c|c|c|c|}
\hline 18 & $\begin{array}{l}\text { Ginger } \\
\text { Gold }\end{array}$ & $\begin{array}{l}\text { yellow- } \\
\text { green }\end{array}$ & high & $\begin{array}{l}\text { fresh } \\
\text { fruit }\end{array}$ & $\begin{array}{l}\text { sweet } \\
\text {-acid }\end{array}$ & $\begin{array}{c}\text { sweet- } \\
\text { acid }\end{array}$ \\
\hline 19 & Gloster 69 & brown & hign & $\begin{array}{l}\text { dried } \\
\text { fruit }\end{array}$ & sweet & sweet \\
\hline 20 & $\begin{array}{l}\text { Gold } \\
\text { Milenium }\end{array}$ & brown & high & $\begin{array}{l}\text { dried } \\
\text { fruit }\end{array}$ & sweet & sweet \\
\hline 21 & Gold Rush & $\begin{array}{l}\text { yellow- } \\
\text { brown }\end{array}$ & high & $\begin{array}{l}\text { fresh } \\
\text { fruit }\end{array}$ & $\begin{array}{l}\text { sweet } \\
\text {-acid }\end{array}$ & $\begin{array}{l}\text { sweet- } \\
\text { acid }\end{array}$ \\
\hline 22 & $\begin{array}{l}\text { Golden } \\
\text { Delicious }\end{array}$ & yellow & high & $\begin{array}{l}\text { fresh } \\
\text { fruit }\end{array}$ & $\begin{array}{l}\text { sweet } \\
\text {-acid }\end{array}$ & $\begin{array}{l}\text { sweet- } \\
\text { acid }\end{array}$ \\
\hline 23 & $\begin{array}{l}\text { Golden } \\
\text { Resistance }\end{array}$ & brown & puree & $\begin{array}{l}\text { dried } \\
\text { fruit }\end{array}$ & $\begin{array}{l}\text { sweet } \\
\text {-acid }\end{array}$ & sweet \\
\hline 24 & $\begin{array}{l}\text { Golden } \\
\text { Smoothee }\end{array}$ & brown & puree & $\begin{array}{l}\text { dried } \\
\text { fruit }\end{array}$ & $\begin{array}{l}\text { sweet } \\
\text {-acid }\end{array}$ & $\begin{array}{c}\text { sweet- } \\
\text { acid }\end{array}$ \\
\hline 25 & $\begin{array}{l}\text { Golden } \\
\text { Winter } \\
\text { Pearmain }\end{array}$ & brown & high & $\begin{array}{l}\text { dried } \\
\text { fruit }\end{array}$ & $\begin{array}{l}\text { sweet } \\
\text {-acid }\end{array}$ & $\begin{array}{c}\text { sweet- } \\
\text { acid }\end{array}$ \\
\hline 26 & Gorana & yellow & hign & $\begin{array}{l}\text { fresh } \\
\text { fruit }\end{array}$ & sweet & sweet \\
\hline 27 & $\begin{array}{l}\text { Granny } \\
\text { Smith }\end{array}$ & $\begin{array}{l}\text { yellow- } \\
\text { green }\end{array}$ & high & $\begin{array}{l}\text { fresh } \\
\text { fruit }\end{array}$ & $\begin{array}{l}\text { acid- } \\
\text { sweet }\end{array}$ & $\begin{array}{l}\text { acid- } \\
\text { sweet }\end{array}$ \\
\hline 28 & $\begin{array}{l}\text { Green } \\
\text { Knyajevska }\end{array}$ & $\begin{array}{l}\text { green- } \\
\text { brown }\end{array}$ & high & $\begin{array}{l}\text { dried } \\
\text { fruit }\end{array}$ & $\begin{array}{l}\text { acid- } \\
\text { sweet }\end{array}$ & $\begin{array}{c}\text { sweet- } \\
\text { acid }\end{array}$ \\
\hline 29 & Hybrid 1/3 & $\begin{array}{l}\text { yellow- } \\
\text { brown }\end{array}$ & high & $\begin{array}{l}\text { dried } \\
\text { fruit }\end{array}$ & $\begin{array}{l}\text { sweet } \\
\text {-acid }\end{array}$ & sweet \\
\hline 30 & $\begin{array}{l}\text { Hybrid } \\
1 / 37\end{array}$ & brown & high & $\begin{array}{l}\text { dried } \\
\text { fruit }\end{array}$ & sweet & $\begin{array}{l}\text { sweet- } \\
\text { acid }\end{array}$ \\
\hline 31 & $\begin{array}{l}\text { Hybrid } \\
2 / 14\end{array}$ & $\begin{array}{l}\text { yellow- } \\
\text { brown }\end{array}$ & high & $\begin{array}{l}\text { fresh } \\
\text { fruit }\end{array}$ & $\begin{array}{l}\text { sweet } \\
\text {-acid }\end{array}$ & $\begin{array}{c}\text { sweet- } \\
\text { acid }\end{array}$ \\
\hline 32 & $\begin{array}{l}\text { Hybrid } \\
2 / 28\end{array}$ & $\begin{array}{l}\text { yellow- } \\
\text { brown }\end{array}$ & puree & $\begin{array}{l}\text { dried } \\
\text { fruit }\end{array}$ & $\begin{array}{l}\text { sweet } \\
\text {-acid }\end{array}$ & $\begin{array}{l}\text { sweet- } \\
\text { acid }\end{array}$ \\
\hline 33 & $\begin{array}{l}\text { Hybrid } \\
2 / 30\end{array}$ & brown & high & $\begin{array}{l}\text { dried } \\
\text { fruit }\end{array}$ & $\begin{array}{l}\text { sweet } \\
\text {-acid }\end{array}$ & sweet \\
\hline 34 & Hybrid 2/4 & brown & puree & $\begin{array}{l}\text { dried } \\
\text { fruit }\end{array}$ & $\begin{array}{l}\text { sweet } \\
\text {-acid }\end{array}$ & sweet \\
\hline 35 & Hybrid 2/8 & brown & puree & $\begin{array}{l}\text { dried } \\
\text { fruit }\end{array}$ & $\begin{array}{l}\text { sweet } \\
\text {-acid }\end{array}$ & $\begin{array}{c}\text { sweet- } \\
\text { acid }\end{array}$ \\
\hline 36 & Hybrid 6 & brown & puree & $\begin{array}{l}\text { dried } \\
\text { fruit }\end{array}$ & $\begin{array}{l}\text { sweet } \\
\text {-acid }\end{array}$ & sweet \\
\hline 37 & Hybrid 7 & $\begin{array}{l}\text { yellow- } \\
\text { brown }\end{array}$ & puree & $\begin{array}{l}\text { dried } \\
\text { fruit }\end{array}$ & $\begin{array}{l}\text { sweet } \\
\text {-acid }\end{array}$ & $\begin{array}{c}\text { sweet- } \\
\text { acid }\end{array}$ \\
\hline 38 & $\begin{array}{l}\text { Hybrid } \\
8 / 22\end{array}$ & brown & puree & $\begin{array}{l}\text { dried } \\
\text { fruit }\end{array}$ & $\begin{array}{l}\text { sweet } \\
\text {-acid }\end{array}$ & $\begin{array}{l}\text { sweet- } \\
\text { acid }\end{array}$ \\
\hline
\end{tabular}




\begin{tabular}{|c|c|c|c|c|c|c|}
\hline 39 & $\begin{array}{l}\text { Hybrid } \\
8 / 35\end{array}$ & $\begin{array}{l}\text { yellow- } \\
\text { brown }\end{array}$ & high & $\begin{array}{l}\text { fresh } \\
\text { fruit }\end{array}$ & $\begin{array}{l}\text { sweet } \\
\text {-acid }\end{array}$ & $\begin{array}{c}\text { sweet- } \\
\text { acid }\end{array}$ \\
\hline 40 & Hybrid 9 & brown & puree & $\begin{array}{l}\text { dried } \\
\text { fruit }\end{array}$ & $\begin{array}{l}\text { sweet } \\
\text {-acid }\end{array}$ & sweet \\
\hline 41 & $\begin{array}{l}\text { Hybrid } \\
9 / 36\end{array}$ & brown & high & $\begin{array}{l}\text { dried } \\
\text { fruit }\end{array}$ & $\begin{array}{l}\text { sweet } \\
\text {-acid }\end{array}$ & $\begin{array}{c}\text { sweet- } \\
\text { acid }\end{array}$ \\
\hline 42 & $\begin{array}{l}\text { Hybrid } \\
\text { Pinova x } \\
\text { Fuji }\end{array}$ & yellow & high & $\begin{array}{l}\text { fresh } \\
\text { fruit }\end{array}$ & $\begin{array}{l}\text { sweet } \\
\text {-acid }\end{array}$ & $\begin{array}{l}\text { acid- } \\
\text { sweet }\end{array}$ \\
\hline 43 & Idagold & brown & high & $\begin{array}{l}\text { dried } \\
\text { fruit }\end{array}$ & $\begin{array}{l}\text { sweet } \\
\text {-acid }\end{array}$ & $\begin{array}{c}\text { sweet- } \\
\text { acid }\end{array}$ \\
\hline 44 & Jonagold & $\begin{array}{l}\text { grenn- } \\
\text { brown }\end{array}$ & high & $\begin{array}{l}\text { dried } \\
\text { fruit }\end{array}$ & $\begin{array}{l}\text { acid- } \\
\text { sweet }\end{array}$ & $\begin{array}{c}\text { sweet- } \\
\text { acid }\end{array}$ \\
\hline 45 & Kadunka & brown & hign & $\begin{array}{l}\text { dried } \\
\text { fruit }\end{array}$ & $\begin{array}{l}\text { sweet } \\
\text {-acid }\end{array}$ & $\begin{array}{c}\text { sweet- } \\
\text { acid }\end{array}$ \\
\hline 46 & $\begin{array}{l}\text { Karastoyan } \\
\text { ka }\end{array}$ & brown & hign & $\begin{array}{l}\text { dried } \\
\text { fruit }\end{array}$ & $\begin{array}{l}\text { acid- } \\
\text { sweet }\end{array}$ & $\begin{array}{l}\text { acid- } \\
\text { sweet }\end{array}$ \\
\hline 47 & $\begin{array}{l}\text { Landsberge } \\
\text { r Renette }\end{array}$ & yellow & high & $\begin{array}{l}\text { fresh } \\
\text { fruit }\end{array}$ & $\begin{array}{l}\text { sweet } \\
\text {-acid }\end{array}$ & $\begin{array}{c}\text { sweet- } \\
\text { acid }\end{array}$ \\
\hline 48 & Liberty & brown & puree & $\begin{array}{l}\text { dried } \\
\text { fruit }\end{array}$ & $\begin{array}{l}\text { sweet } \\
\text {-acid }\end{array}$ & $\begin{array}{c}\text { sweet- } \\
\text { acid }\end{array}$ \\
\hline 49 & Marlena & brown & puree & $\begin{array}{l}\text { dried } \\
\text { fruit }\end{array}$ & $\begin{array}{l}\text { sweet } \\
\text {-acid }\end{array}$ & $\begin{array}{c}\text { sweet- } \\
\text { acid }\end{array}$ \\
\hline 50 & Martinika & yellow & hign & $\begin{array}{l}\text { fresh } \\
\text { fruit }\end{array}$ & $\begin{array}{l}\text { sweet } \\
\text {-acid }\end{array}$ & $\begin{array}{c}\text { sweet- } \\
\text { acid }\end{array}$ \\
\hline 51 & Melrose & brown & high & $\begin{array}{l}\text { dried } \\
\text { fruit }\end{array}$ & $\begin{array}{l}\text { sweet } \\
\text {-acid }\end{array}$ & $\begin{array}{l}\text { acid- } \\
\text { sweet }\end{array}$ \\
\hline 52 & $\begin{array}{l}\text { Mollie's } \\
\text { Delicious }\end{array}$ & brown & high & $\begin{array}{l}\text { dried } \\
\text { fruit }\end{array}$ & $\begin{array}{l}\text { sweet } \\
\text {-acid }\end{array}$ & $\begin{array}{c}\text { sweet- } \\
\text { acid }\end{array}$ \\
\hline 53 & Mutsu & $\begin{array}{l}\text { green- } \\
\text { brown }\end{array}$ & high & $\begin{array}{l}\text { dried } \\
\text { fruit }\end{array}$ & $\begin{array}{l}\text { sweet } \\
\text {-acid }\end{array}$ & sweet \\
\hline 54 & $\begin{array}{l}\text { Ovcha } \\
\text { Mutsuna }\end{array}$ & $\begin{array}{l}\text { yellow- } \\
\text { green }\end{array}$ & high & $\begin{array}{l}\text { fresh } \\
\text { fruit }\end{array}$ & $\begin{array}{l}\text { sweet } \\
\text {-acid }\end{array}$ & $\begin{array}{c}\text { sweet- } \\
\text { acid }\end{array}$ \\
\hline 55 & $\begin{array}{l}\text { Pasific } \\
\text { Rose }\end{array}$ & yellow & hign & $\begin{array}{l}\text { fresh } \\
\text { fruit }\end{array}$ & sweet & sweet \\
\hline 56 & Pink Lady & brown & high & $\begin{array}{l}\text { dried } \\
\text { fruit }\end{array}$ & $\begin{array}{l}\text { acid- } \\
\text { sweet }\end{array}$ & $\begin{array}{c}\text { sweet- } \\
\text { acid }\end{array}$ \\
\hline 57 & Pinova & brown & high & $\begin{array}{l}\text { dried } \\
\text { fruit }\end{array}$ & $\begin{array}{l}\text { sweet } \\
\text {-acid }\end{array}$ & $\begin{array}{c}\text { sweet- } \\
\text { acid }\end{array}$ \\
\hline 58 & Priam & brown & hign & $\begin{array}{l}\text { dried } \\
\text { fruit }\end{array}$ & sweet & sweet \\
\hline 59 & Prima & brown & puree & $\begin{array}{l}\text { dried } \\
\text { fruit }\end{array}$ & $\begin{array}{l}\text { sweet } \\
\text {-acid }\end{array}$ & $\begin{array}{c}\text { sweet- } \\
\text { acid }\end{array}$ \\
\hline
\end{tabular}

\begin{tabular}{|c|c|c|c|c|c|c|}
\hline 60 & Reandra & brown & high & $\begin{array}{l}\text { dried } \\
\text { fruit }\end{array}$ & $\begin{array}{l}\text { sweet } \\
\text {-acid }\end{array}$ & $\begin{array}{l}\text { acid- } \\
\text { sweet }\end{array}$ \\
\hline 61 & Red Chief & brown & puree & $\begin{array}{l}\text { dried } \\
\text { fruit }\end{array}$ & sweet & sweet \\
\hline 62 & $\begin{array}{l}\text { Red } \\
\text { Delicios }\end{array}$ & brown & puree & $\begin{array}{l}\text { dried } \\
\text { fruit }\end{array}$ & sweet & sweet \\
\hline 63 & $\begin{array}{l}\text { Reinette du } \\
\text { Canada }\end{array}$ & brown & puree & $\begin{array}{l}\text { dried } \\
\text { fruit }\end{array}$ & $\begin{array}{l}\text { acid- } \\
\text { sweet }\end{array}$ & $\begin{array}{l}\text { acid- } \\
\text { sweet }\end{array}$ \\
\hline 64 & Renora & yellow & high & $\begin{array}{l}\text { fresh } \\
\text { fruit }\end{array}$ & $\begin{array}{l}\text { sweet } \\
\text {-acid }\end{array}$ & $\begin{array}{l}\text { sweet- } \\
\text { acid }\end{array}$ \\
\hline 65 & Rosana & $\begin{array}{l}\text { brown- } \\
\text { green }\end{array}$ & high & $\begin{array}{l}\text { dried } \\
\text { fruit }\end{array}$ & $\begin{array}{l}\text { sweet } \\
\text {-acid }\end{array}$ & sweet \\
\hline 66 & Rosemary & brown & hign & $\begin{array}{l}\text { dried } \\
\text { fruit }\end{array}$ & sweet & sweet \\
\hline 67 & Rubinola & yellow & hign & $\begin{array}{l}\text { fresh } \\
\text { fruit }\end{array}$ & sweet & sweet \\
\hline 68 & Sansa & yellow & hign & $\begin{array}{l}\text { fresh } \\
\text { fruit }\end{array}$ & $\begin{array}{l}\text { acid- } \\
\text { sweet }\end{array}$ & sweet \\
\hline 69 & Sekai Ichi & brown & hign & $\begin{array}{l}\text { dried } \\
\text { fruit }\end{array}$ & sweet & sweet \\
\hline 70 & Siyana & yellow & puree & $\begin{array}{l}\text { fresh } \\
\text { fruit }\end{array}$ & sweet & sweet \\
\hline 71 & Skrinyanka & brown & hign & $\begin{array}{l}\text { dried } \\
\text { fruit }\end{array}$ & $\begin{array}{l}\text { acid- } \\
\text { sweet }\end{array}$ & $\begin{array}{c}\text { sweet- } \\
\text { acid }\end{array}$ \\
\hline 72 & $\begin{array}{l}\text { Super } \\
\text { Chief }\end{array}$ & brown & hign & $\begin{array}{l}\text { dried } \\
\text { fruit }\end{array}$ & $\begin{array}{l}\text { sweet } \\
\text {-acid }\end{array}$ & $\begin{array}{c}\text { sweet- } \\
\text { acid }\end{array}$ \\
\hline 73 & Teser T219 & yellow & hign & $\begin{array}{l}\text { fresh } \\
\text { fruit }\end{array}$ & $\begin{array}{l}\text { sweet } \\
\text {-acid }\end{array}$ & sweet \\
\hline 74 & Tetovka & brown & high & $\begin{array}{l}\text { dried } \\
\text { fruit }\end{array}$ & $\begin{array}{l}\text { sweet } \\
\text {-acid }\end{array}$ & $\begin{array}{l}\text { acid- } \\
\text { sweet }\end{array}$ \\
\hline 75 & Topaz & brown & puree & $\begin{array}{l}\text { dried } \\
\text { fruit }\end{array}$ & sweet & sweet \\
\hline 76 & Tsiganka & brown & puree & $\begin{array}{l}\text { dried } \\
\text { fruit }\end{array}$ & $\begin{array}{l}\text { acid- } \\
\text { sweet }\end{array}$ & $\begin{array}{c}\text { sweet- } \\
\text { acid }\end{array}$ \\
\hline 77 & Wellington & $\begin{array}{l}\text { light } \\
\text { green }\end{array}$ & hign & $\begin{array}{l}\text { fresh } \\
\text { fruit }\end{array}$ & acid & acid \\
\hline 78 & $\begin{array}{l}\text { White } \\
\text { Kandile }\end{array}$ & brown & hign & $\begin{array}{l}\text { dried } \\
\text { fruit }\end{array}$ & sweet & sweet \\
\hline 79 & $\begin{array}{l}\text { White } \\
\text { Winter } \\
\text { Calville }\end{array}$ & brown & hign & $\begin{array}{l}\text { dried } \\
\text { fruit }\end{array}$ & $\begin{array}{l}\text { sweet } \\
\text {-acid }\end{array}$ & $\begin{array}{c}\text { sweet- } \\
\text { acid }\end{array}$ \\
\hline 80 & $\begin{array}{l}\text { Wild apple } \\
\text { plant }\end{array}$ & yellow & high & $\begin{array}{l}\text { fresh } \\
\text { fruit }\end{array}$ & acid & acid \\
\hline 81 & $\begin{array}{l}\text { Winter } \\
\text { Lemon }\end{array}$ & brown & puree & $\begin{array}{l}\text { dried } \\
\text { fruit }\end{array}$ & $\begin{array}{l}\text { sweet } \\
\text {-acid }\end{array}$ & $\begin{array}{c}\text { sweet- } \\
\text { acid }\end{array}$ \\
\hline 82 & Wiygik & $\begin{array}{l}\text { brown- } \\
\text { green }\end{array}$ & high & $\begin{array}{l}\text { dried } \\
\text { fruit }\end{array}$ & $\begin{array}{l}\text { sweet } \\
\text {-acid }\end{array}$ & acid \\
\hline
\end{tabular}




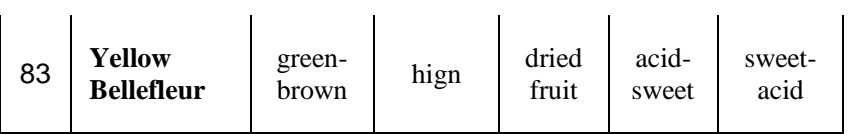

Table 4 Ratios between Electroconductivity (EC), Total Dissolved Solids (TDS) and Salt.

\begin{tabular}{|c|c|c|c|c|c|c|}
\hline № & $\begin{array}{r}\text { Juice } \\
\text { parameters }\end{array}$ & 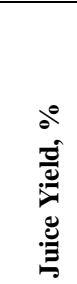 & 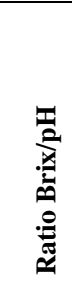 & 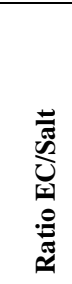 & 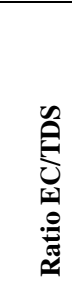 & 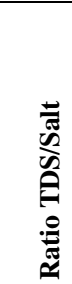 \\
\hline 1 & Aivanija & 4.00 & 2.25 & 1.99 & 1.50 & 1.33 \\
\hline 2 & Winter Banana & 3.00 & 5.36 & 1.96 & 1.50 & 1.31 \\
\hline 3 & Belgolden & 16.00 & 3.30 & 1.97 & 1.49 & 1.32 \\
\hline 4 & $\begin{array}{l}\text { Berner } \\
\text { Rosenapfel }\end{array}$ & 3.00 & 2.30 & 1.85 & 1.49 & 1.23 \\
\hline 5 & Besapara & 13.70 & 3.36 & 1.91 & 1.36 & 1.41 \\
\hline 6 & Braeburn & 10.26 & 3.15 & 1.94 & 1.48 & 1.31 \\
\hline 7 & Buhavitsa & 53.00 & 4.23 & 1.98 & 1.49 & 1.33 \\
\hline 8 & Charden & 41.66 & 4.04 & 1.89 & 1.49 & 1.27 \\
\hline 9 & COOP 10 & 3.00 & 2.70 & 2.06 & 1.50 & 1.37 \\
\hline 10 & Defloga & 70.73 & 3.21 & 2.01 & 1.49 & 1.34 \\
\hline 11 & Democrat & 56.32 & 3.59 & 1.50 & 1.97 & 1.08 \\
\hline 12 & Elegia & 5.00 & 4.02 & 1.19 & 2.24 & 1.88 \\
\hline 13 & Erwin Bauer & 60.00 & 4.81 & 2.01 & 1.49 & 1.34 \\
\hline 14 & Florina & 61.61 & 4.06 & 1.99 & 1.49 & 1.34 \\
\hline 15 & Free Redstar & 5.00 & 2.67 & 2.07 & 1.50 & 1.38 \\
\hline 16 & Freedom & 61.02 & 4.14 & 1.96 & 1.50 & 1.30 \\
\hline 17 & Gala Mondial & 51.60 & 2.86 & 1.94 & 1.50 & 1.30 \\
\hline 18 & Ginger Gold & 77.50 & 3.84 & 1.99 & 1.49 & 1.34 \\
\hline 19 & Gloster 69 & 38.50 & 2.89 & 1.98 & 1.50 & 1.32 \\
\hline 20 & Gold Milenium & 63.44 & 3.69 & 1.99 & 1.50 & 1.33 \\
\hline 21 & Gold Rush & 71.00 & 3.91 & 1.97 & 1.50 & 1.32 \\
\hline 22 & Golden Delicious & 66.51 & 3.47 & 1.98 & 1.49 & 1.33 \\
\hline
\end{tabular}

\begin{tabular}{|c|c|c|c|c|c|c|}
\hline 23 & $\begin{array}{l}\text { Golden } \\
\text { Resistance }\end{array}$ & 43.60 & 4.55 & 2.07 & 1.49 & 1.39 \\
\hline 24 & $\begin{array}{l}\text { Golden } \\
\text { Smoothee }\end{array}$ & 30.00 & 3.75 & 1.97 & 1.49 & 1.32 \\
\hline 25 & $\begin{array}{l}\text { Golden Winter } \\
\text { Pearmain }\end{array}$ & 76.50 & 3.94 & 1.98 & 1.49 & 1.32 \\
\hline 26 & Gorana & 30.82 & 4.59 & 1.50 & 1.85 & 0.81 \\
\hline 27 & Granny Smith & 72.50 & 3.91 & 3.34 & 1.82 & 1.83 \\
\hline 28 & $\begin{array}{l}\text { Green } \\
\text { Knyajevska }\end{array}$ & 56.50 & 4.03 & 1.97 & 1.49 & 1.32 \\
\hline 29 & Hybrid 1/3 & 51.76 & 3.96 & 2.01 & 1.50 & 1.34 \\
\hline 30 & Hybrid 1/37 & 54.67 & 2.82 & 1.97 & 1.49 & 1.32 \\
\hline 31 & Hybrid 2/14 & 70.00 & 4.26 & 1.98 & 1.51 & 1.32 \\
\hline 32 & Hybrid 2/28 & 7.26 & 3.70 & 1.97 & 1.49 & 1.32 \\
\hline 33 & Hybrid 2/30 & 23.00 & 3.41 & 1.37 & 0.66 & 2.07 \\
\hline 34 & Hybrid 2/4 & 42.00 & 4.33 & 1.86 & 1.49 & 1.25 \\
\hline 35 & Hybrid 2/8 & 20.00 & 3.71 & 2.02 & 1.49 & 1.35 \\
\hline 36 & Hybrid 6 & 34.17 & 3.24 & 1.87 & 1.49 & 1.25 \\
\hline 37 & Hybrid 7 & 21.28 & 3.47 & 1.97 & 1.49 & 1.32 \\
\hline 38 & Hybrid 8/22 & 18.93 & 4.21 & 1.96 & 1.49 & 1.32 \\
\hline 39 & Hybrid 8/35 & 61.99 & 4.86 & 2.00 & 1.49 & 1.35 \\
\hline 40 & Hybrid 9 & 19.82 & 4.27 & 1.98 & 1.50 & 1.33 \\
\hline 41 & Hybrid 9/36 & 42.98 & 3.60 & 1.88 & 1.50 & 1.25 \\
\hline 42 & $\begin{array}{l}\text { Hybrid Pinova x } \\
\text { Fuji }\end{array}$ & 49.50 & 3.53 & 1.97 & 1.49 & 1.32 \\
\hline 43 & Idagold & 49.57 & 3.98 & 2.03 & 1.49 & 1.36 \\
\hline 44 & Jonagold & 59.90 & 5.19 & 2.06 & 1.49 & 1.38 \\
\hline 45 & Kadunka & 43.72 & 3.80 & 1.86 & 1.49 & 1.25 \\
\hline 46 & Karastoyanka & 67.00 & 3.75 & 2.04 & 1.51 & 1.35 \\
\hline 47 & $\begin{array}{l}\text { Landsberger } \\
\text { Renette }\end{array}$ & 63.87 & 4.79 & 1.93 & 1.50 & 1.29 \\
\hline 48 & Liberty & 17.67 & 4.37 & 2.23 & 1.49 & 1.49 \\
\hline 49 & Marlena & 16.34 & 3.31 & 1.96 & 1.49 & 1.31 \\
\hline
\end{tabular}




\begin{tabular}{|c|c|c|c|c|c|c|}
\hline 50 & Martinika & 55.94 & 4.34 & 1.98 & 1.50 & 1.32 \\
\hline 51 & Melrose & 72.22 & 3.11 & 1.98 & 1.48 & 1.34 \\
\hline 52 & $\begin{array}{l}\text { Mollie's } \\
\text { Delicious }\end{array}$ & 75.51 & 4.25 & 1.96 & 1.51 & 1.30 \\
\hline 53 & Mutsu & 70.00 & 3.64 & 2.05 & 1.49 & 1.38 \\
\hline 54 & Ovcha Mutsuna & 63.00 & 5.28 & 2.06 & 1.50 & 1.38 \\
\hline 55 & Pasific Rose & 58.34 & 3.58 & 2.03 & 1.49 & 1.36 \\
\hline 56 & Pink Lady & 61.20 & 4.53 & 2.05 & 1.50 & 1.37 \\
\hline 57 & Pinova & 64.65 & 3.99 & 1.97 & 1.49 & 1.32 \\
\hline 58 & Priam & 38.92 & 1.98 & 1.96 & 1.50 & 1.31 \\
\hline 59 & Prima & 20.00 & 3.44 & 1.52 & 1.09 & 1.39 \\
\hline 60 & Reandra & 69.77 & 4.01 & 2.01 & 1.48 & 1.36 \\
\hline 61 & Red Chief & 13.22 & 2.48 & 2.00 & 1.49 & 1.34 \\
\hline 62 & Red Delicios & 15.30 & 3.08 & 1.95 & 1.49 & 1.31 \\
\hline 63 & $\begin{array}{l}\text { Reinette du } \\
\text { Canada }\end{array}$ & 9.39 & 3.75 & 1.87 & 1.49 & 1.25 \\
\hline 64 & Renora & 75.68 & 4.44 & 1.92 & 1.50 & 1.28 \\
\hline 65 & Rosana & 61.68 & 2.99 & 1.99 & 1.49 & 1.33 \\
\hline 66 & Rosemary & 33.00 & 3.23 & 1.96 & 1.50 & 1.31 \\
\hline 67 & Rubinola & 46.22 & 3.74 & 2.02 & 1.49 & 1.36 \\
\hline 68 & Sansa & 32.00 & 3.36 & 2.00 & 1.49 & 1.34 \\
\hline 69 & Sekai Ichi & 54.00 & 3.80 & 1.93 & 1.50 & 1.29 \\
\hline 70 & Siyana & 9.80 & 4.11 & 2.10 & 1.51 & 1.39 \\
\hline 71 & Skrinyanka & 27.30 & 3.94 & 1.98 & 1.54 & 1.28 \\
\hline 72 & Super Chief & 30.00 & 2.59 & 1.99 & 1.50 & 1.32 \\
\hline 73 & Teser T219 & 76.57 & 3.52 & 1.85 & 1.44 & 1.28 \\
\hline 74 & Tetovka & 59.00 & 3.38 & 2.00 & 1.49 & 1.34 \\
\hline 75 & Topaz & 15.92 & 4.11 & 1.95 & 1.49 & 1.31 \\
\hline 76 & Tsiganka & 29.73 & 4.49 & 1.99 & 1.49 & 1.33 \\
\hline 77 & Wellington & 64.00 & 3.75 & 2.44 & 1.61 & 1.52 \\
\hline 78 & White Kandile & 42.52 & 3.66 & 1.85 & 1.50 & 1.24 \\
\hline 79 & $\begin{array}{l}\text { White Winter } \\
\text { Calville }\end{array}$ & 41.95 & 4.25 & 1.86 & 1.49 & 1.24 \\
\hline 80 & Wild apple plant & 66.66 & 4.43 & 1.94 & 1.45 & 1.34 \\
\hline 80 & Winter Lemon & 5.00 & 2.93 & 1.85 & 1.49 & 1.24 \\
\hline
\end{tabular}

\begin{tabular}{|l|l|c|c|c|c|c|}
81 & Wiygik & 65.00 & 3.50 & 2.01 & 1.49 & 1.34 \\
\hline 82 & Yellow Bellefleur & 61.50 & 3.76 & 1.85 & 1.54 & 1.20 \\
\hline
\end{tabular}

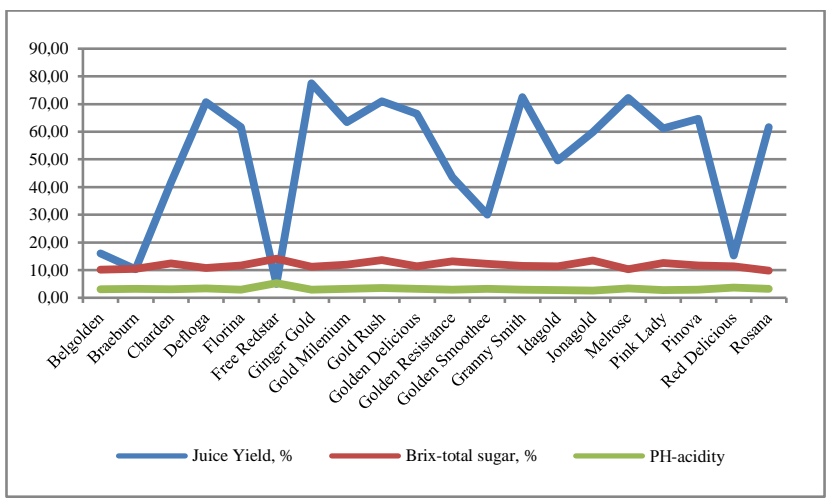

Fig. 1 Diagram of the measured technological parameters of the juices and purees, according to the variety

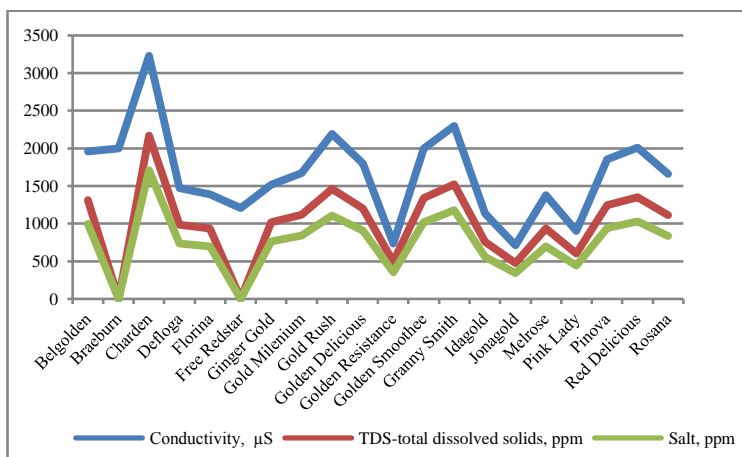

Fig. 2 Diagram of the measured technological parameters of the juices and purees, according to the variety

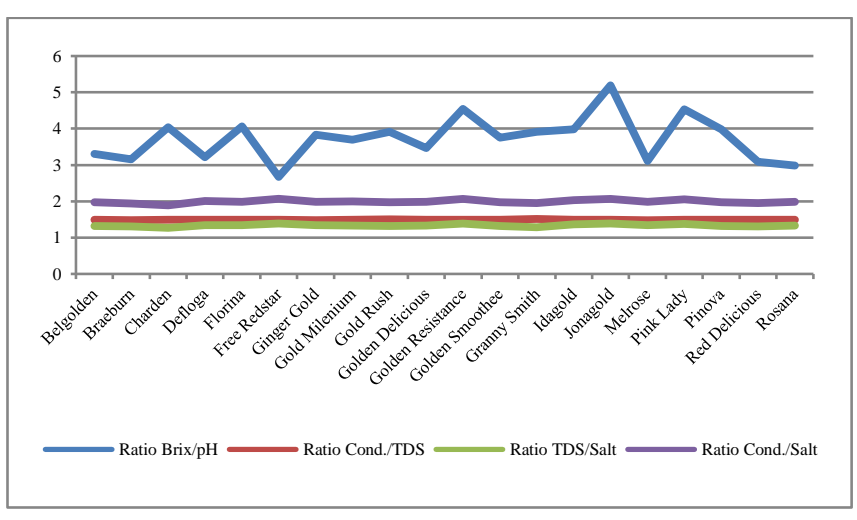

Fig. 3 Diagram of the ratios between total sugar content (Brix), total acidity $(\mathrm{pH})$, Conductivity 
(Cond.), total dissolved solids (TDS), and total salt content (Salt).

\section{Discussion}

Table 1 presents the results of the measurements of the ecological parameters of apples and their juices. All tested apple varieties and their juices have low radioactivity in accordance with the regulations. The natural common radiation background for the Kyustendil region, Bulgaria, according to the Bulgarian Civil Protection Service, is $0,16 \mu \mathrm{Sv} / \mathrm{h}$ (as it fluctuates from 0,10 to $0,18 \mu \mathrm{Sv} / \mathrm{h}$ ), and is less often outside these norms. To some extent, there is a relationship between the common radiation background and the radioactivity of the fruit. With few exceptions, the radioactivity of the juices directly depends on the radioactivity of the fruits, with a slight decrease in the values in the juice in most cases.

The sulfates $\mathrm{SO}_{4}{ }^{2-}$ in the juice of all apple varieties, vary between 200 and $400 \mathrm{mg} / \mathrm{l}$ or about $300 \mathrm{mg} / \mathrm{l}$ average. The measured values are minimal, according to the scope of the applied test, and the sulfates are probably part of the organic and mineral substance of the apples, and it is not a result of plant protection or environmental pollution of the area.

Sulfites $\mathrm{SO}_{3}{ }^{2-}$, lead $\mathrm{Pb}$, arsenic As, nitrates $\mathrm{NO}_{3}{ }^{-}$and nitrites $\mathrm{NO}_{2}^{-}$are not detected by the applied test methods.

Manganese (Mn) and zinc ( $\mathrm{Zn}$ ) have constant contents, because of this reason it is assumed that they are also part of the natural chemical composition of apples, and not the result of environmental pollution. Manganese content in apples of $2,37 \mathrm{mg} / 100 \mathrm{~g}$ and zinc $0,88 \mathrm{mg} / 100 \mathrm{~g}$ have been reported by [15], ie. the contents of these two elements measured by in present study $(\mathrm{Mn}=2$ $\mathrm{mg} / \mathrm{l}$ and $\mathrm{Zn}=1 \mathrm{mg} / \mathrm{l}$ ) are part of the nutrition composition of apples and cannot be claimed for ecological pollution.

In conclusion, it can be said that in agroecological view, the studied apple varieties are of high quality and ecologically clean.

Tables 2,3,4 show that the highest yields (over $70 \%$ juice) separate apples of the varieties Defloga, Ginger Gold, Gold Rush, Granny Smith and Melrose. Juice with color and taste of fresh fruit give the varieties Florina, Free Redstar, Ginger Gold, Gold Rush, Golden Delicious and Granny Smith. Some varieties of apples do not produce juice by the method of cold pressing, as well as fruit pulp for fruit flour, but they produce high quality fruit puree, such as varieties Belgolden, Breburn, Charden and Red Delicious.

As a result of the study, it was found that there are stable relationships between electrical conductivity, total dissolved solids and total salt content in all tested juices, respectively: Cond./TDS 1,5; TDS/Salt 1,3; Cond./Salt 2 .

The ratio between the total sugars (Brix) and the total acidity $(\mathrm{pH})$ varies from 3 to 5 . The most tasty, delicious and with the best color (fresh yellow or greenish-yellow) are the juices which $\mathrm{Brix} / \mathrm{pH}$ ratio is 4 .

The glucose content of the tested juices exceeds the maximum range of the apparatus used (> 33,3 mmol/l), with an exception of Florina juice, in which the glucose is $29,6 \mathrm{mmol} / \mathrm{l}$ and the juices by some hybrids. It has been found that the ratio of fructose to glucose in several studied varieties varies between 2.1 and $3.4[14,25]$. Therefore, the lower glucose content of this variety also implies a lower fructose content. The fructose/glucose ratio can be used as an indicator of the authenticity of apple juice, as a ratio of 1,6 being the lowest expected and respectively the maximum is of 6,09, with average value of 3,03 for typical apple juice [18]. Therefore, from the studied apples, the Florina variety can be recommended for the production of apple foods for diabetics.

It is assumed that apples with the highest values of electrical conductivity of the juice have the most favorable effect on the human body in terms of its detoxification, due to the high mobility and ability to bind and migrate the electrolyte elements. In this regard, the most useful varieties are Breburn, Charden, Gold Rush, Golden Smoothie, Granny Smith and Red Delicious.

\section{Conclusion}

The highest yields of juice (over 70\%) separate apples from the varieties Defloga, Ginger Gold, Gold Rush, Granny Smith and Melrose. Juice with good color and taste of fresh fruit give the varieties Florina, Free Redstar, Ginger Gold, Gold Rush, Golden Delicious and Granny Smith. Some varieties of apples do not produce juice by the method of cold pressing, as well as fruit pulp for fruit flour, but they produce high quality fruit puree, such as varieties Belgolden, Breburn, Charden and Red Delicious.

Variety for diabetics is Florina and Hybrid 9/36, Hybrid 2/28, and Hybrid 2/30. The lowest glucose content was measured for it, respectively 
29,$6 ; 25,3 ; 26,7 ; 27,1 \mathrm{mmol} / 1$ or it means lower fructose content.

Varieties with the strongest ability to remove toxins from the body are those with the most electrolytes: Braburn, Charden, Gold Rush, Golden Smoothie, Granny Smith and Red Delicious. Based on sensory analysis, it was found that the most delicious and best-colored juices and purees are obtained from the varieties Charden, Florina, Ginger Gold, Gold Millennium, Gold Rush, Golden Smoothie, Granny Smith, Ida Gold and Pinova, where the $\mathrm{Brix} / \mathrm{pH}$ ratio is 4 .

There are stable ratios between electrical conductivity, total dissolved solids and total salts in all tested juices, respectively: Cond./TDS $=1,5$; TDS/Salt=1,3; Cond./Salt=2.

One of the best varieties for the production of $100 \%$ apple juice by the cold pressing method is the Granny Smith variety, which participates in almost all positive indicators of juice quality.

\section{Acknowledgements}

The research and publication were supported by the Bulgarian Ministry of Education and Science (Fund "Scientific Research") - Project "Breeding and application on new yellow-green crisp apple in Bulgaria and China" - Contract: № KP-06-China /4 from 20.12.2018.

\section{References:}

1. Narvaiz, P., P., N. LuciaKaupert. (1988). Preservation of apples by irradiation, Food Chemistry, Vol. 27, Issue 4, 1988, Pages 273-281.

2. Al-Bachir, M. (1999). Effect of gamma irradiation on storability of apples (Malus domestica L.), Plant Foods for Human Nutrition, vol. 54, pages1-11(1999).

3. Thomas, B. (1998). Quality of apples following gamma irradiation and cold storage. Journal International Journal of Food Sciences and Nutrition, Volume 49, 1998 - Issue 6, Pages 485-492.

4. Dolchinkov, N. (2017). Radiation Background of the Atmosphere, Soil and Water in Bulgaria and Its Monitoring in the Contemporary Political Conditions. Conference: Technics, technologies, education, safety 2017, Veliko Tarnovo, Bulgaria, Volume: 1.

5. Dimitrova, S., D., Sotirov. (2015). Breeding Activities and Cultivar
Composition of Apple (Malus domestica L.). Plant Science, Vol. LII, 2, 52-58.

6. Dzuvinov, V., Gandev, S., Arnaudov, V., Rankova, Z., Nacheva, L., Dobrevska, G., Apple, Biofruit Ltd., Bulgaria, 2016.

7. B. Turkan, A.S. Canbolat, A.B. Etemoglu (2018). 3-D simulation of simultaneous heat and mass transfer of apple. Bulgarian Chemical Communications, Volume 50, Special Issue G (pp. 215 - 224) 2018.

8. Massaguer, P., Silva, A., Chaves, R., Gressoni, I., Jr. (2014). Fruits and Vegetables/Fruit and Vegetable Juices, Encyclopedia of Food Microbiology (Second Edition), pp. 992-999.

9. Ryan, R. (2014). Safety of Food and Beverages: Soft Drinks and Fruit Juices, Encyclopedia of Analytical Science (2nd edition) Encyclopedia of food safety, Vol. 3, pp. 360-363.

10. Sadecka, J., Polonsky, J. (2005). Food and Nutritional Analysis/Soft Drinks, Encyclopedia of Analytical Science (2nd edition), pp. 272-279.

11. Celik, F., Gundogdu, M., Alp, S., Muradoglu, F., Ercişli, S., Gecer, M., Canan, I. (2017). Determination of Phenolic Compounds, Antioxidant Capacity and Organic Acids Contents of Prunus domestica L., Prunus cerasifera Ehrh. and Prunus spinosa L. Fruits by HPLC, Acta Chromatographica, Vol. 29, (4), pp. 1-4.

12. Rafe Hagee, Brandi Benedict, Kristina Simpkins, Dalton Anderson, and Nicholas Sink. (2015). Aple juice portfolio. South WestVirginia Governer's School, 13 pp.

13. Seiiedlou, S., H.R. Ghasemzadeh, N. Hamdami, F. Talati and M. Moghaddam. (2010). Convective drying of apple: mathematical modeling and determination of some quality parameters. Int. J. Agric. Biol., 12: 171-178.

14. Katharina Hermann, Ursula BordewickDell. (2018). Fructose in different apple varieties Implications for apple consumption in persons affected by fructose intolerance. Science \& Research/Original Contribution, 48 Ernaehrungs Umschau International, Vol. 3/2018, 48-52.

15. Isabelle Kasongo Omba, Criss Koba Mjumbe, Guyslain Mashini Ngongo, Oscar Luboya Numbi. (2020). Quality Control of Juices Produced in Democratic Republic of 
Congo and Marketed in Lubumbashi. Food and Nutrition Sciences, 11, 255-261.

16. Gheorghe CAMPEANU, Gabriela NEATA, Gina DARJANSCHI. (2009). Chemical Composition of the Fruits of Several Apple Cultivars Growth as Biological Crop, Not. Bot. Hort. Agrobot. Cluj 37 (2), 161-164.

17. Thomas A. Eiselea, Stephen R. Drakeb. (2005). The partial compositional characteristics of apple juice from 175 apple varieties. Journal of Food Composition and Analysis, 18 (2005) 213221.

18. Yinrong Lu, L. Yeap Foo. (1998). Constitution of some chemical components of apple seed. Food Chemistry, Elsevier, Vol. 61, No. 1/2, pp. 29-33, 1998.

19. Włodarska, K., Pawlak-Lemańska, K., Górecki, T., Sikorska, E.. (2017). Classification of commercial apple juices based on multivariate analysis of their chemical profiles, International Journal of Food Properties, Vol. 20, № 8, 2017, pp. 1773-1785.

20. Khan, S., Iqbal, Z., Ubairah, Khan, A., Shah, F., Shinwari, A., (2017). Nutritional and Microbial Evaluation of Commercial Apple Juices Available in Market of Peshawar City, Global Journal of Medical Research: L Nutrition \& Food Science, Vol. 17, № 1, 2017, pp. 16-20.

21. Campeanu, G., Neata, G., Darjanschi, G. (2009). Chemical Composition of the Fruits of Several Apple Varieties Growth as Biological Crop, Notulae Botanicae Horti Agrobotanici Cluj-Napoca, Vol. 37, № 2, 2009, pp. 161-164.

22. Persic, M., Mikulic-Petkovsek, M., Slatnar, A., Veberic, R. (2017). Chemical composition of apple fruit, juice and pomace and the correlation between phenolic content, enzymatic activity and browning, Elsevier, LWT - Food Science and Technology, Vol. 82, 2017, pp. 23-31.

23. Onivogui, G., Zhang, H., Mlyuka, E., Diaby, M., Song, Y. (2014). Chemical Composition, Nutritional Properties and Antioxidant Activity of Monkey Apple (Anisophyllea laurina R. Br. ex Sabine), Journal of Food and Nutrition Research, Vol. 2, № 6, 2014, pp. 281-287.

24. Eiselea, T., Drakeb, S. (2005). The partial compositional characteristics of apple juice from 175 apple varieties, Elsevier, Journal of Food Composition and Analysis, Vol. 18, 2005, 213-221.

25. Hermann K, Bordewick-Dell, U., Fructose in different apple. Implications for apple consumption in persons affected by fructose intolerance, Science \& Research, Ernahrungs Umschau, Vol. 65, № 3, 2017, pp. $48-52$.

26. Sotirov, A., Glavev, N., Sotirov, D. Dimitrova, S., Pistalov, N., Sotirov, V., Sotirov, K., Vezenkova, R., Andonova, R., Velinova, L. (2020). Characteristics of apple juices, produced by the cold-pressure method. Proceedings Youth Forums "Science, Technology, Innovation, Business-2020, Plovdiv, Bulgaria, pp. 8388.

\section{Creative Commons Attribution License 4.0 (Attribution 4.0 International, CC BY 4.0)}

This article is published under the terms of the Creative Commons Attribution License 4.0 https://creativecommons.org/licenses/by/4.0/deed.en_US 